Article

\title{
New Biocompatible Polyurethanes: Synthesis, Structural Characterization and Cytotoxicity
}

\author{
Barbara S. Gregorí Valdés ${ }^{1,3}$, Clara S. B. Gomes ${ }^{2}$, Pedro T. Gomes ${ }^{2, *}$, José R. Ascenso ${ }^{2, *}$, \\ Hermínio P. Diogo ${ }^{2}$, Lídia M. Gonçalves ${ }^{3}$, Rui Galhano dos Santos ${ }^{1}$, Helena M. Ribeiro ${ }^{3}$ and \\ João C. Bordado 1,* \\ 1 CERENA, Departamento de Engenharia Química, Instituto Superior Técnico, Universidade de Lisboa, Av. \\ Rovisco Pais,1049-001 Lisboa, Portugal \\ 2 Centro de Química Estrutural, Departamento de Engenharia Química, Instituto Superior Técnico, \\ Universidade de Lisboa, Av. Rovisco Pais, 1049-001 Lisboa, Portugal \\ 3 Research Institute for Medicine and Pharmaceutical Science (iMed.ULisboa), Faculty of Pharmacy, \\ Universidade de Lisboa, Av. Prof. Gama Pinto, 1649-003 Lisboa, Portugal \\ * Correspondence: pedro.t.gomes@tecnico.ulisboa.pt, jose.ascenso@tecnico.ulisboa.pt, \\ jcbordado@tecnico.ulisboa.pt;
}

\begin{abstract}
The synthesis of four samples of new polyurethanes was evaluated by changing the ratio of the diol monomers used, poly(propylene glycol) (PPG) and D-isosorbide, in the presence of aliphatic isocyanates such as the isophorone diisocyanate (IPDI) and 4,4'-methylenebis(cyclohexyl isocyanate) (HMDI). The thermal properties of the four polymers obtained were determined by DSC, exhibiting $\mathrm{T}_{\mathrm{g}}$ values in the range $55-70{ }^{\circ} \mathrm{C}$, and their molecular structure characterized by FTIR, ${ }^{1} \mathrm{H}$ and ${ }^{13} \mathrm{C}$ NMR spectroscopies. The diffusion coefficients of these polymers in solution were measured by the Pulse Gradient Spin Echo (PGSE) NMR method, enabling the calculation of the corresponding hydrodynamic radii in diluted solution $(1.62-2.65 \mathrm{~nm})$. The molecular weights were determined by GPC/SEC and compared with the values determined by quantitative ${ }^{13} \mathrm{C}$ NMR analysis. Finally, the biocompatibility of the polyurethanes was assessed using the HaCaT keratinocyte cell line by the MTT reduction assay method showing values superior to $70 \%$ cell viability.
\end{abstract}

Keywords: Biocompatibility; GPC/SEC; Keratinocyte cells; NMR; Polyurethane; Renewable sources

\section{Introduction}

Polyurethanes (PUs) are extremely versatile polymers, due to their easy structural tunability and to their elastomeric and thermoplastic behavior, their syntheses from the combination of new monomers being extensively studied by several groups [1-6].

PU materials are usually composed of two types of phases, in which hard glassy phase-enriched domains are dispersed in a matrix of soft rubbery segments. These hard segments contribute to the modulus, mechanical strength and elevated temperature properties owing to the strong intermolecular interactions such as hydrogen bonding among urethane groups, whereas the soft segments afford elasticity and low-temperature mechanical properties [2]. Moreover, the properties of PUs are remarkably affected by the content, type, and molecular weight of the soft segments. Therefore, the choice of monomers used to synthesize PUs is dependent on the final applications of the materials [7]. For example, monomers offering excellent durability are selected for polymers to be used for prostheses [8], those increasing the compatibility of PU with tissues are applied for pacemakers [9], and monomers offering thermal stability are carefully chosen for catheters that require sterilization processes $[7,10]$. 
We have been interested in the synthesis of linear polyurethanes from carbohydrates suitably functionalized for applications in the pharmaceutical industry, namely for the use in nail lacquer formulations, as they are being considered as a solution to deliver relevant drugs to infected nails [11]. However, the currently available polymer excipients present some disadvantages for the formulation of vehicles for an efficient drug delivery using therapeutic nail lacquers. Isosorbide is a monomer already employed in the synthesis of biocompatible polymers $[3,7,12,13]$. This glycol is a chiral and quite thermostable diol, which raises the $T_{\mathrm{g}}$ of the corresponding polymers, and can be obtained by reduction of glucose followed by dehydration (Figure 1). On the other hand, the poly(propylene glycol) (PPG) is a biocompatible polyether that has been extensively investigated for<smiles>O=CC(O)C(O)C(O)C(O)CO</smiles>

Figure 1. Two step procedure for the synthesis of isosorbide from D-glucose [14].

application as a biomedical material conferring elastomeric properties to polyurethanes [15-17]. This monomer was then chosen as a second glycol in the outlined synthesis. To avoid the use of monomers that probably yield toxic compounds, the isophorone diisocyanate (IPDI) and 4,4'-methylenebis(cyclohexyl isocyanate) (HMDI) were envisaged as options to access nontoxic formulations, since those isocyanates have already been used in the synthesis of several biocompatible polyurethanes [16,18,19].

The present work describes the synthesis of new polyurethanes from D-isosorbide by step growth polymerization, involving diisocyanates, such as IPDI or HDMI, and PPG as soft segments, and the characterization of their chemical structures, molecular weights and thermal properties by Fourier-Transform Infrared (FTIR) and ${ }^{1} \mathrm{H}$ and ${ }^{13} \mathrm{C}$ Nuclear Magnetic Resonance spectroscopies (NMR), Gel Permeation Chromatography/Size-Exclusion Chromatography (GPC/SEC) and Differential Scanning Calorimetry (DSC). The novelty of this work consists in the synthesis and characterization of new polyurethanes biocompatible with keratinocyte cells that can participate, as drug delivery supports, in the formulation of nail lacquers used in the treatment of onychomycosis.

\section{Experimental Section}

\subsection{Materials}

The D-isosorbide was provided by Acrös, HMDI by Bayer Material Science, and IPDI and 2,2'-dimorpholinyldiethylether (DMDEE) by Sigma-Aldrich. PPG (VORANOL 1010L) was supplied by Dow Chemical. Anhydrous ethanol was purchased from Carlo Erba. DMSO- $d_{6}, \mathrm{CDCl}_{3}$, and tetramethylsilane (TMS) $(99.9 \%$ purity) used in NMR determinations were acquired from Sigma-Aldrich. The HPLC grade tetrahydrofuran (THF) and the polystyrene (PS) standards used in GPC/SEC were obtained from Aldrich and TSK Tosoh Co., respectively.

\subsection{Polymer Synthesis}

The polyurethanes reported in the present work were synthesized in two steps. The first one involved in the quasi-prepolymerization method by reacting the monomers IPDI or HMDI and PPG for ca. 3 hours, at $80^{\circ} \mathrm{C}$. Subsequently, the second step comprised the reaction of the previously afforded quasi-prepolymers with $\mathrm{D}$-isosorbide diol monomer, for 1 hour, at $80^{\circ} \mathrm{C}$. 
Three polyurethane quasi-prepolymers were prepared by reacting IPDI or HMDI and PPG in a $250 \mathrm{~mL}$ glass flask, under a dry nitrogen atmosphere, to prevent and avoid the presence of moisture and subsequently the formation of urea bonds during the synthesis. The reaction took place with the dropwise addition of PPG to the quasi-prepolymer, under mechanical stirring ( $400 \mathrm{rpm}$ ), at $80{ }^{\circ} \mathrm{C}$ and in the presence of $0.5 \mathrm{~mL}$ of the catalyst DMDEE. The reactions were practically completed in 3 hours, as determined by the isocyanate (-NCO) groups content existing in the reactions mixtures (determined by back titration with an excess of $\mathrm{N}, \mathrm{N}$-dibutylamine with standard $\mathrm{HCl}[20]$ ). Viscometry measurements of the reaction mixtures by cone-plate rheometry were alos used to evaluate the course of the reaction [21] (using a shear stress-controlled ICI Cone and Plate rheometer - London, LTD - at $25^{\circ} \mathrm{C}$ and $20 \mathrm{~Hz}$, using parallel plates, with an upper plate diameter of $20 \mathrm{~mm}$, with a gap of $0.4 \mathrm{~mm}$ ). The molar ratios of the monomers used in each synthesized pre-polymer are presented in Table 1.

Table 1. Molar ratios of monomers employed in the synthesis of the quasi-prepolymers.

\begin{tabular}{cccc}
\hline Monomers & Pre-1 & Pre-2 & Pre-3 \\
\hline IPDI & 6 & 6 & - \\
HMDI & - & - & 6 \\
PPG & 1 & 2 & 1 \\
\hline
\end{tabular}

\subsubsection{Syntheses of polyurethanes PU1-PU4}

The syntheses of the four polyurethanes PU1-PU4 were carried out in a $250 \mathrm{~mL}$ reactor by the addition of an appropriate amount of D-isosorbide to the IPD/PPG or HMDI/PPG polyurethane quasi-prepolymers obtained previously (see section 2.2.1.), according to predetermined molar ratio of monomers and prepolymer, which are presented in Table 2 . The reactions took place in $1 \mathrm{~h}$, at 80 ${ }^{\circ} \mathrm{C}$, under a dry nitrogen atmosphere.

Table 2. Molar ratios of monomers and prepolymer employed in the synthesis of the polyurethanes PU1-PU4.

\begin{tabular}{ccccc}
\hline \multirow{2}{*}{ Monomers } & \multicolumn{4}{c}{ Polymers } \\
\cline { 2 - 5 } & PU1 & PU2 & PU3 & PU4 \\
\hline IPDI & 6 & 6 & 6 & - \\
HMDI & - & - & - & 6 \\
PPG & 1 & 2 & 1 & 1 \\
D-isosorbide & 5 & 5 & 6 & 5 \\
\hline
\end{tabular}

\subsection{FTIR characterization}

The quasi-prepolymers' FTIR spectra were acquired with a Nexus Thermo Nicolet spectrometer equipped with attenuated total reflectance (ATR) device for the quasi-prepolymers. The solid samples of polyurethane (approximately of $1 \mathrm{mg}$ ) were finely powdered and dispersed in a $\mathrm{KBr}$ matrix (200 mg). For each polymer, a pellet was then formed by compressing the sample at $784 \mathrm{MPa}$. The FTIR spectra were obtained at room temperature, in the range of $4000 \mathrm{~cm}^{-1}$ to $600 \mathrm{~cm}^{-1}$, after 128 scans with a resolution of $4 \mathrm{~cm}^{-1}$.

\subsection{NMR spectroscopy characterization}

${ }^{1} \mathrm{H}$ and ${ }^{13} \mathrm{C}$ NMR spectra were obtained using a Bruker AVANCE III $500 \mathrm{MHz}$ spectrometer equipped with a $5 \mathrm{~mm}$ BBO probe. To prepare each of the polyurethanes samples 20-53 $\mathrm{mg}$ of the polymers were dissolved in $0.6 \mathrm{~mL}$ of DMSO- $d_{6}$. Quantitative ${ }^{13} \mathrm{C}$ NMR spectra were obtained under inverse gated decoupling, with a delay of 9s and a 80 degree pulse. 2D COSY, HSQC and HMBC 
spectra were obtained with Bruker standard sequences. All the spectra were run at room temperature employing TMS as an internal standard.

\subsection{Measurement of the diffusion coefficients by NMR spectroscopy}

The Pulse Gradient Spin Echo (PGSE) method is a relatively simple technique of measuring diffusion coefficients in solution. It combines NMR spin echoes with pulsed-field gradients of varible strength. To determine the diffusion coefficient of a molecule in solution, a series of ${ }^{1} \mathrm{H}$ spectra is obtained in which the strengh of the gradients is increased and the attenuation of the intensity of the proton peaks due to diffusion is monitored [22]. In our study, the diffusion coefficient (D) of each polymer was determined at five different concentrations. For this purpose a series of five solutions was prepared with concentrations $0.03 \%, 0.07 \%, 0.15 \%, 0.3 \%$ and $1 \%(\mathrm{w} / \mathrm{v})$ in DMSO- $d_{6}$. The diffusion coefficients of the polymers at infinite diffusion $\left(\mathrm{D}_{0}\right)$ were obtained from plots of D versus concentration (Tables S1 to S4 and Figure S12 in the Supplementary Material). The $\mathrm{D}$ values were measured using the PGSE method in a NMR Bruker AVANCE III $500 \mathrm{MHz}$ spectrometer with a $3 \mathrm{~mm}$ BBO probe and a z-gradient shielded coil. This combination gives a maximum possible gradient of $0.56 \mathrm{~T} \mathrm{~m}^{-1}$. A bipolar stimulated echo sequence (STE) with smoothed square gradients and WATERGATE solvent suppression were used [23]. The signal intensity was monitored as a function of the square of the gradient amplitude and the resulting self-diffusion coefficients D were calculated according to the echo attenuation equation for STE:

$$
\mathrm{I}=\mathrm{I}_{0} \exp \left[-\mathrm{D}(\gamma \delta \mathrm{g})^{2}(\Delta-\delta / 3)\right]
$$

where $I_{0}$ is the intensity in the absence of gradient pulses, $\delta$ is the duration of the applied gradient, $\gamma$ is the gyromagnetic ratio of the nucleus and $\Delta$ is the diffusion time. For each polymer, the areas of three or four single proton peaks were used in the fittings and the average D value was taken. For more details see the Supplementary Material.

The solutions of the polymers were transferred to $3 \mathrm{~mm}$ NMR tubes to a total volume of $0.3 \mathrm{~mL}$. To guarantee reproducibility of the results this volume was kept constant in all the samples. The temperature was controlled at $30^{\circ} \mathrm{C}$ by a BCU05 Bruker unit with an air flow of $521 \mathrm{Lh}^{-1}$.

\subsection{Gel Permeation Chromatography/Size-exclusion Chromatography (GPC/SEC) characterization}

The analyses were made in a HPLC Waters chromatograph containing a Waters 515 isocratic pump and a Waters 2414 refractive index detector. In this apparatus, the oven was stabilized at 35 ${ }^{\circ} \mathrm{C}$, and the elution of samples was carried out through two PolyPore columns (Polymer Labs), protected by a PolyPore Guard column (Polymer Labs). The software Empower ${ }^{\circledR}$ performed the acquisition and data processing.

THF was used as eluent, at a flow rate of $1.0 \mathrm{~mL} \cdot \mathrm{min}^{-1}$. Before use, the solvent was filtered through $0.45 \mu \mathrm{m}$ PTFE membranes Fluoropore (Millipore or Pall Corporation) and degassed in an ultrasound bath for $45 \mathrm{~min}$. The oligomer/polymer samples were also filtered across $0.20 \mu \mathrm{m}$ PTFE filters Durapore (Millipore). Molecular weights were calibrated relative to polystyrene standards (TSK Tosoh Co.). As a result, it should be taken into account that small deviations could occur when the present polymer samples were analyzed.

\subsection{Thermal behavior characterization by Differential Scanning Calorimetry (DSC)}

The characterization of the thermal behavior of the polymer samples was performed with a 2920 MDSC system from TA Instruments Inc., equipped with a refrigerated cooling accessory (LNCA), which provided automatic and continuous programmed sample cooling down to $123 \mathrm{~K}$.

The temperature scale of the instrument was calibrated with five standard compounds and the heat flow scale was calibrated with indium and tin. Further details regarding the calibration process can be found in the literature [24]. The samples were accurately weighed $( \pm 0.1 \mu \mathrm{g})$ in aluminum pans on a Mettler UMT2 ultra-micro balance in air. All the measurements were performed under dry high purity helium gas (Air Liquide N55), at a flow rate of $30 \mathrm{~mL} \mathrm{~min}^{-1}$. 
The protocol used in the DSC measurements consisted of two consecutive identical thermal cycles: samples were cooled to $-110^{\circ} \mathrm{C}$ and then heated until $230^{\circ} \mathrm{C}$, at $10 \mathrm{~K} \mathrm{~min}^{-1}$.

\subsection{In vitro cytotoxicity assay}

The polyurethanes were completely dissolved in ethanol, under stirring (200 rpm), at a concentration of $250 \mathrm{mg} \mathrm{mL}^{-1}$. Later, one side of the $10 \mathrm{~mm}$ diameter glass coverslip was expared with this solution and dried for $30 \mathrm{~min}$.

The biocompatibility of the polymer was evaluated in vitro by direct contact with cells, following the ISO 10993-5:2009 recommendation guidelines [25]. The procedure used was a previously published [11].

The data are expressed as the mean and the respective standard deviation (mean \pm SD) of 6 experiments. The statistical evaluation of data was performed using one-way analysis of variance (ANOVA). The Tukey-Kramer multiple comparison tests (GraphPad PRISM 5 software, USA), were used to compare the significance of the difference between the groups; a $p<0.05$ was accepted as statistically significant.

\section{Results and discussion}

\subsection{Syntheses of the PUs}

A family of aliphatic biocompatible PUs, based on IPDI or HDMI, D-isosorbide and PPG, were synthesized in order to convey the active principle. The PUs were designed to be composed of various ratios of soft segments (PPG based blocks) and hard segments (D-isosorbide based blocks). A two stage step growth polymerization method was employed. In an early step, the pre-polymerization of an excess of a diisocyanate monomer (IPDI or HMDI) with the PPG diol, catalyzed by DMDEE, was carried out by stirring the reaction mixture for 3 hours, at $80{ }^{\circ} \mathrm{C}$. Then, a complementary step consisted
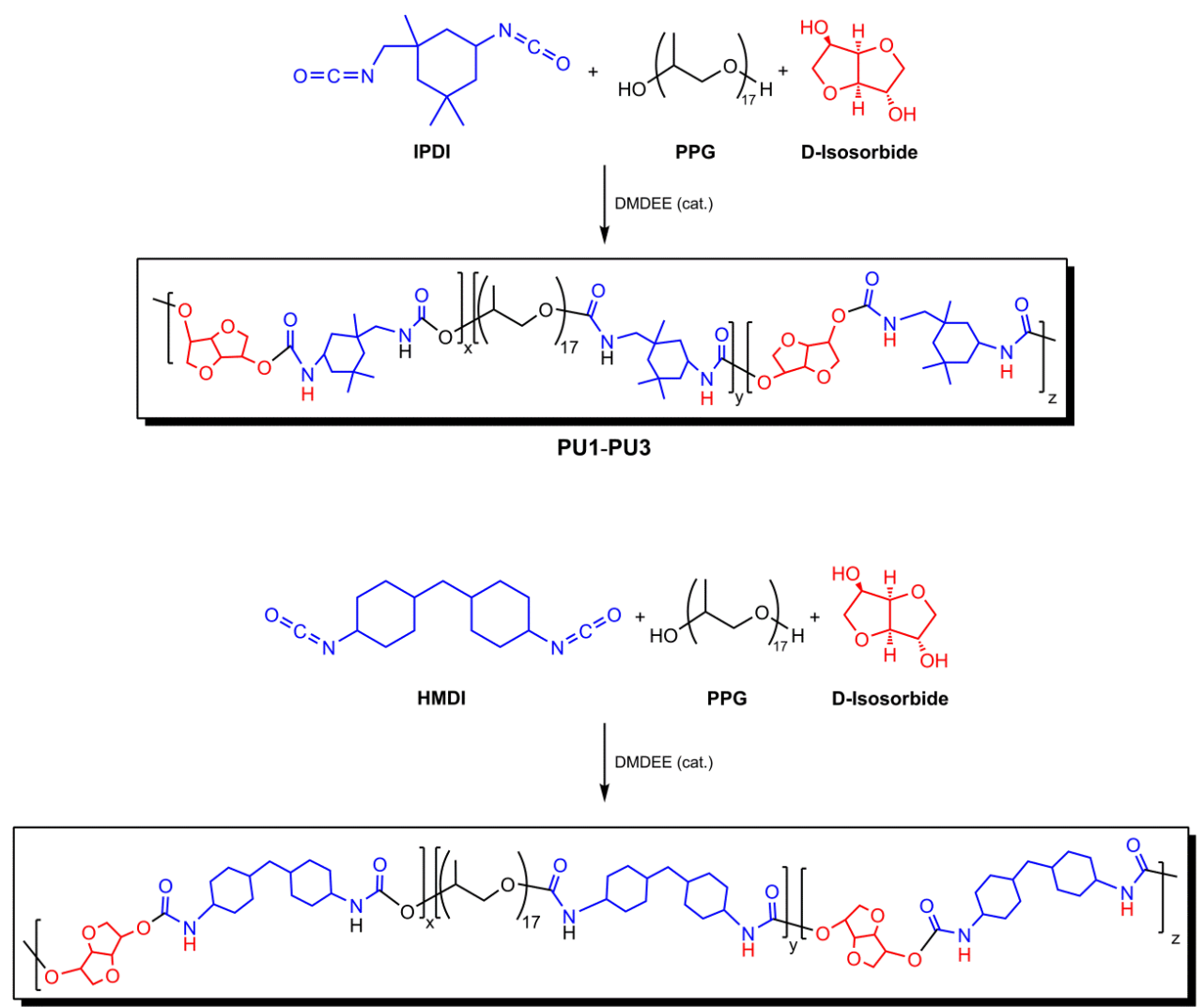

PU4

Figure 2. Synthesis of polyurethanes PU1-PU4. 
in the addition of a pre-defined amount of the D-isosorbide diol monomer to the previous pre-polymer reaction mixtures (catalyst included), at the abovementioned temperature, for an additional hour. The polyurethanes PU1-PU3 were obtained from the reaction of IPDI, PPG, and D-isosorbide, according to the ratios listed in Table 2, whereas PU4 was obtained from the reaction of HMDI, PPG, and D-isosorbide (Figure 2). The four PU products obtained in these reactions were characterized by FTIR, NMR and GPC/SEC without further purification.

\subsection{FTIR of the PUs}

The FTIR spectra of the three quasi-prepolymers, which were synthesized according to the monomer molar ratios defined in Table 1, are presented in Figure 3. In all the quasi-prepolymers a band at $2252 \mathrm{~cm}^{-1}$ is observed, indicating the presence of isocyanate groups (-NCO) corresponding to the monomers IPDI and HMDI, which is in agreement with the reports of the literature (2270 to 2250 $\mathrm{cm}^{-1}$ for the $\mathrm{N}=\mathrm{C}=\mathrm{O}$ stretching vibration) [26-28]. The spectra of the quasi-prepolymers also showed bands at $2927 \mathrm{~cm}^{-1}$, corresponding to $-\mathrm{CH}_{2}$ - groups, and the peak of the ether group at $1527 \mathrm{~cm}^{-1}$. These bands correspond to PPG segments (pure PPG presents a $-\mathrm{CH}_{2}$ - band between $2970-2850 \mathrm{~cm}^{-1}$ and another one corresponding to the ether group at $1526 \mathrm{~cm}^{-1}$ [29]). The band corresponding to the carbonyl groups is observed between 1677-1708 $\mathrm{cm}^{-1}$, which is indicative of the condensation reaction between the -OH group of PPG and the -NCO groups of HMDI and IPDI [30-32].

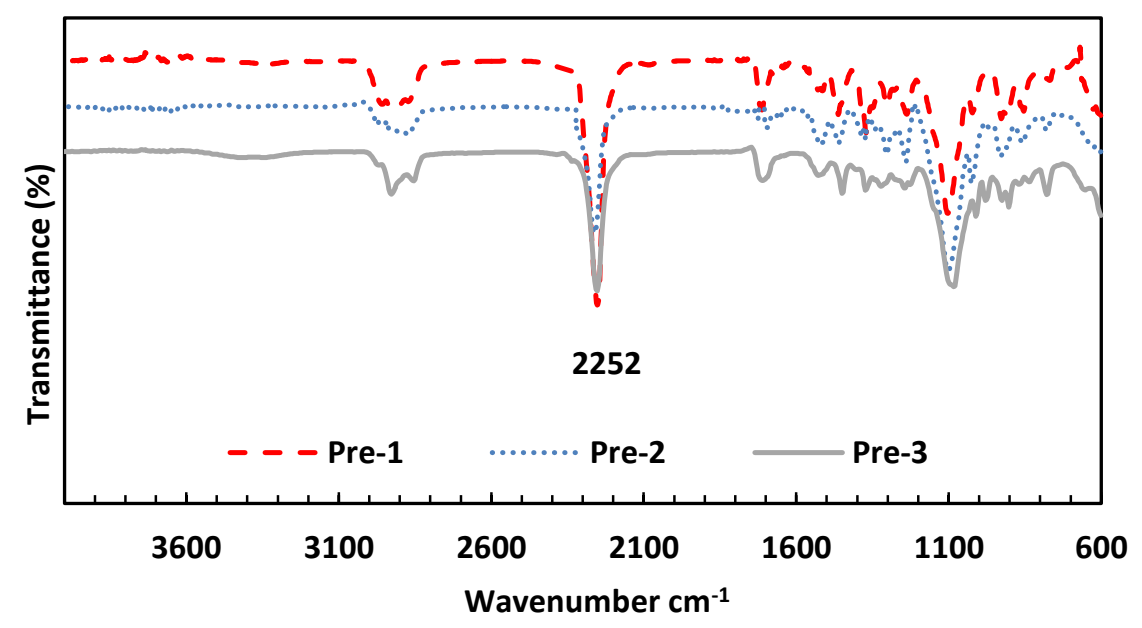

Figure 3. FTIR spectra of the three polyurethane quasi-prepolymers.

The FTIR spectra of the resulting IPDI based polyurethanes PU1-PU3, and corresponding monomers are shown in Figure 4. The characteristic band of the isosorbide monomer -OH groups is observed at $3366 \mathrm{~cm}^{-1}$, whereas the band at $2973 \mathrm{~cm}^{-1}$ corresponds to the isosorbide methylene group $\left(-\mathrm{CH}_{2}-\right)$ [33]. In the PUs spectra, a broad signal between $3700-3200 \mathrm{~cm}^{-1}$ remains. Such signal likely corresponds to the superimposition of $-\mathrm{NH}$ - stretching vibrations of urethane groups of the PUs hard segments as well as to terminal isosorbide $-\mathrm{OH}$ groups and terminal urea $-\mathrm{NH}_{2}$ groups (the latter resulting from the hydrolysis of the PUs' former -NCO urethane terminal groups upon exposure to air after the reaction). The - $\mathrm{CH}_{2}$ - bands, identified in isosorbide and PPG, are detected in the spectra of the PUs between $3050-2800 \mathrm{~cm}^{-1}$. The hard segments carbonyl bands can be observed between 1705-1691 $\mathrm{cm}^{-1}[30,34]$. The disappearance of $-\mathrm{N}=\mathrm{C}=\mathrm{O}$ stretching vibration, around 2250 $\mathrm{cm}^{-1}$, in the spectra of the synthesized polyurethanes suggests that there is no unreacted isocyanate groups [19], i.e. all the isocyanate groups reacted with the monomers -OH groups (and the remaining terminal ones with $\mathrm{H}_{2} \mathrm{O}$ from moisture) [35]. 


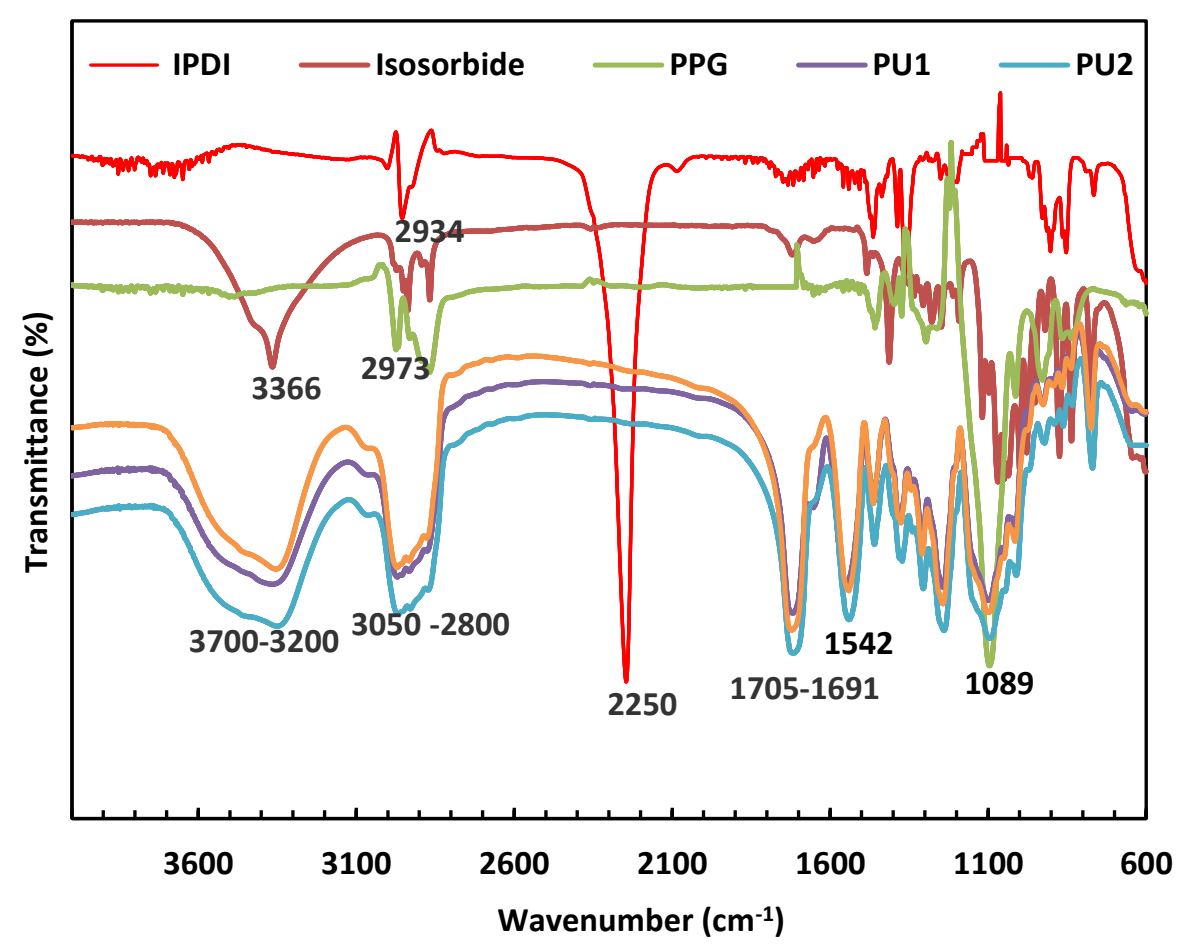

Figure. 4. FTIR spectra of the monomers Isosorbide, PPG, IPDI, and of the polyurethanes PU1-PU3.

On the other hand, the -NH- bending bands of the synthesized PUs were identified at $1542 \mathrm{~cm}^{-1}$ (Figure 4) [27]. These bands were observed in the reaction between polycaprolactone and 2-isocyanate ethylmethacrylate [36]. The - $\mathrm{NH}$ - bending of the urethane group was also detected by da Silva et al. at the same wavenumber [37]. Furthermore, the C-O-C stretching was observed at 1089 $\mathrm{cm}^{-1}[34]$.

The FTIR spectrum of the HMDI based polyurethane PU4 is depicted in Figure 5. In this spectrum, the - $\mathrm{CH}_{2}$ - stretching bands (from PPG) are observed between 2927 and $2854 \mathrm{~cm}^{-1}$, the band of the urethane carbonyl being detected at $1704 \mathrm{~cm}^{-1}$. The $\mathrm{C}-\mathrm{N}$ stretching bands, combined with those of the bending of N-H in the plane, are typically observed at $1523 \mathrm{~cm}^{-1}$ and $1446 \mathrm{~cm}^{-1}$, demonstrating the occurrence of the reaction between the hydroxyl group and the isocyanate [38]. The C-O-C characteristic band was identified at $1079 \mathrm{~cm}^{-1}$.

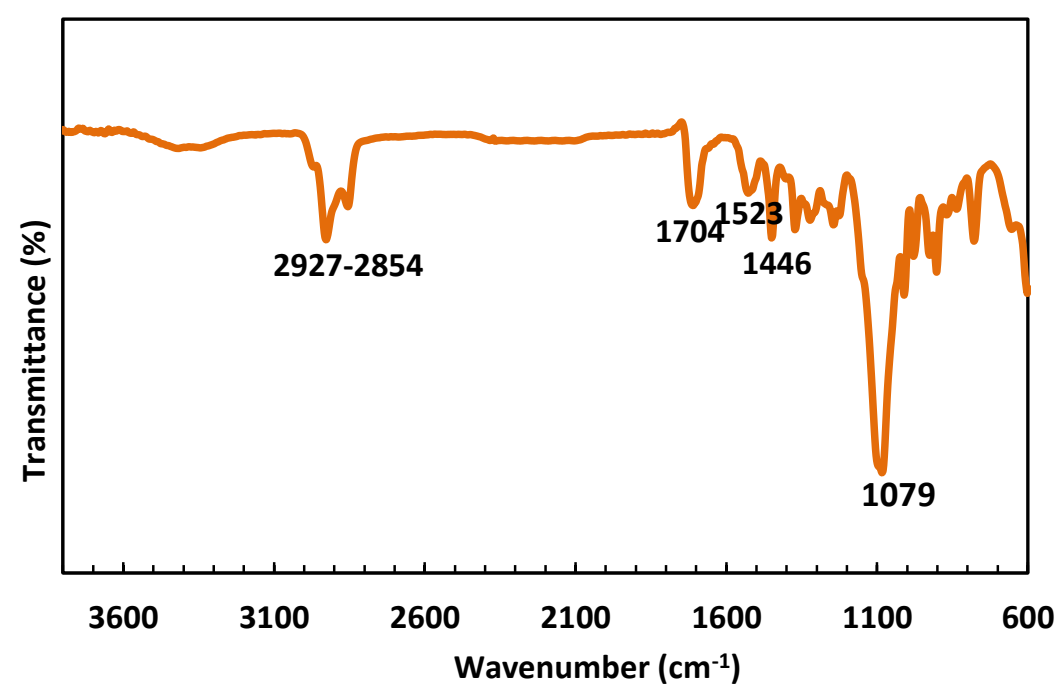


Figure 5. FTIR spectrum of the polyurethane PU4.

\subsection{NMR of the PUs}

The ${ }^{1} \mathrm{H}$ NMR spectra of polyurethanes PU1, PU2 and PU3 are shown in Figure 6.

The presence of amide protons near to $7.0 \mathrm{ppm}$ of the urethane groups-OCONH -indicates that the reaction between the isosorbide hydroxyl groups and the pre-polymer isocyanate groups has occurred. In the research published by Besse et al., the characteristic - $\mathrm{NH}$ - of the urethane group was identified at 7.1 ppm as a result of the synthesis of polyhydroxyurethanes by reaction of isosorbide dicyclocarbonate with four commercial diamines [3]. Other authors mentioned that the proton from the -OCONH- group, obtained from the reaction between IPDI and a polyester, can also be observed

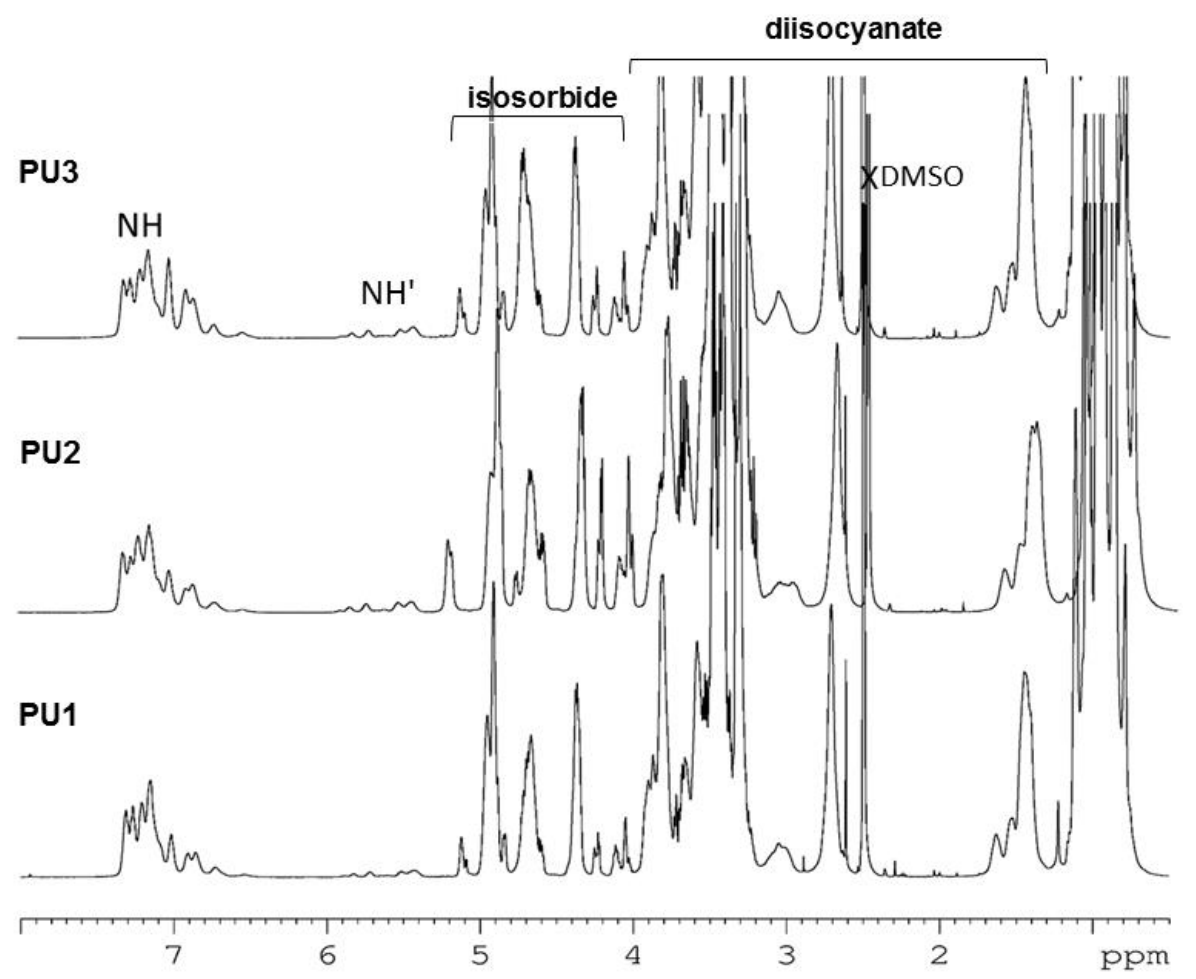

Figure 6. ${ }^{1} \mathrm{H}$ NMR spectra of PU1, PU2 and PU3 in DMSO- $d 6$. Protons of terminal groups are primed.

at $6.8,6.82$ and $7.2 \mathrm{ppm}$ [39]. Literature data shows data in the polyurethanes resulting from the reaction between isosorbide, hexamethylene diiosocyanate (HDI) and poly(tetramethylene glycol) (PTMG), the -NH- peak is described at $8.07 \mathrm{ppm}$ [7]. The ${ }^{1} \mathrm{H}$ NMR spectra of the in-chain isosorbide units, peaks from 4.0-5.2 ppm, of the IPDI units, peaks from 2.6-4.0 ppm, and of the IPDI methyl groups, ca. $1.0 \mathrm{ppm}$, were assigned on the basis of the existing literature of pre-polymers made from the reaction of PPG with IPDI monomers [17]. The assignment of the isosorbide and IPDI resonances in the spectra of PU1-PU3 were further confirmed by means of 2D NMR experiments (COSY, HSQC and HMBC). Some of these spectra are shown in Figures S1 and S3-S7 of the Supplementary Material. The peaks of the ${ }^{1} \mathrm{H}$ NMR spectra of the PPG soft-segment are observed as multiple resonances between 3.2-4.0 ppm for both the $\mathrm{CH}$ and $\mathrm{CH}_{2}$ protons, and in the range 1.0-1.2 ppm for the methyl groups. The ${ }^{1} \mathrm{H}$ NMR spectrum of PU4 is presented in Figure 7. The peaks ca. 7.2 ppm in Figure 7 correspond to the $\mathrm{NH}$ of urethane groups. The signals at 4.0-5.2 ppm are an evidence for the presence of isosorbide groups in the structure of polyurethane [18]. The strong peaks centered at 1.2 and $3.4 \mathrm{ppm}$ belong to the $\mathrm{CH}_{3}$ and $\mathrm{CH}+\mathrm{CH}_{2}$ protons of the PPG segment, respectively. The peaks characteristic of HMDI units are identified at 0.75-3.6 ppm [31,40]. The assignment of the spectra of the isosorbide and HMDI units were further confirmed by means of 2D NMR experiments (HSQC and $\mathrm{HMBC}$ ). They are shown in Figures S8-S11 of the Supplementary Material. The unreacted 
hydroxyl in exo position of the isosorbide end group appears as a minor resonance at ca. $5.1 \mathrm{ppm}$ and is characteristic of polyurethanes with isosorbide as a chain extender [19].

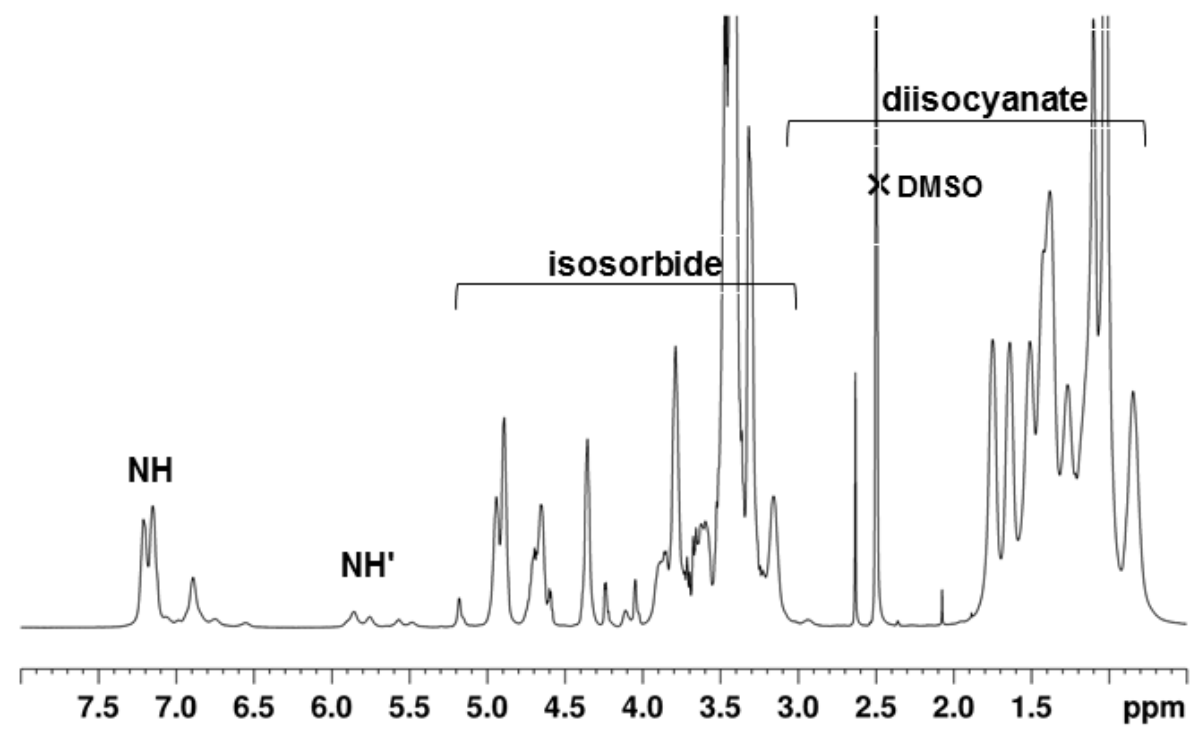

Figure 7. ${ }^{1} \mathrm{H}$ NMR spectrum of PU4 in DMSO- $d 6$. Protons of terminal groups are primed.

The assignment of the ${ }^{13} \mathrm{C}$ NMR spectra of polyurethanes PU1, PU2, and PU3 presented in Figure 8 was complicated owing to the fact that monomer IPDI is available as a mixture of cis and trans isomers (3:1), and we had to use HSQC and HMBC spectra to fully assign the in-chain carbons (see Figures S2 and S4-S7 of the Supplementary Material). The peaks from the isosorbide units are obser-

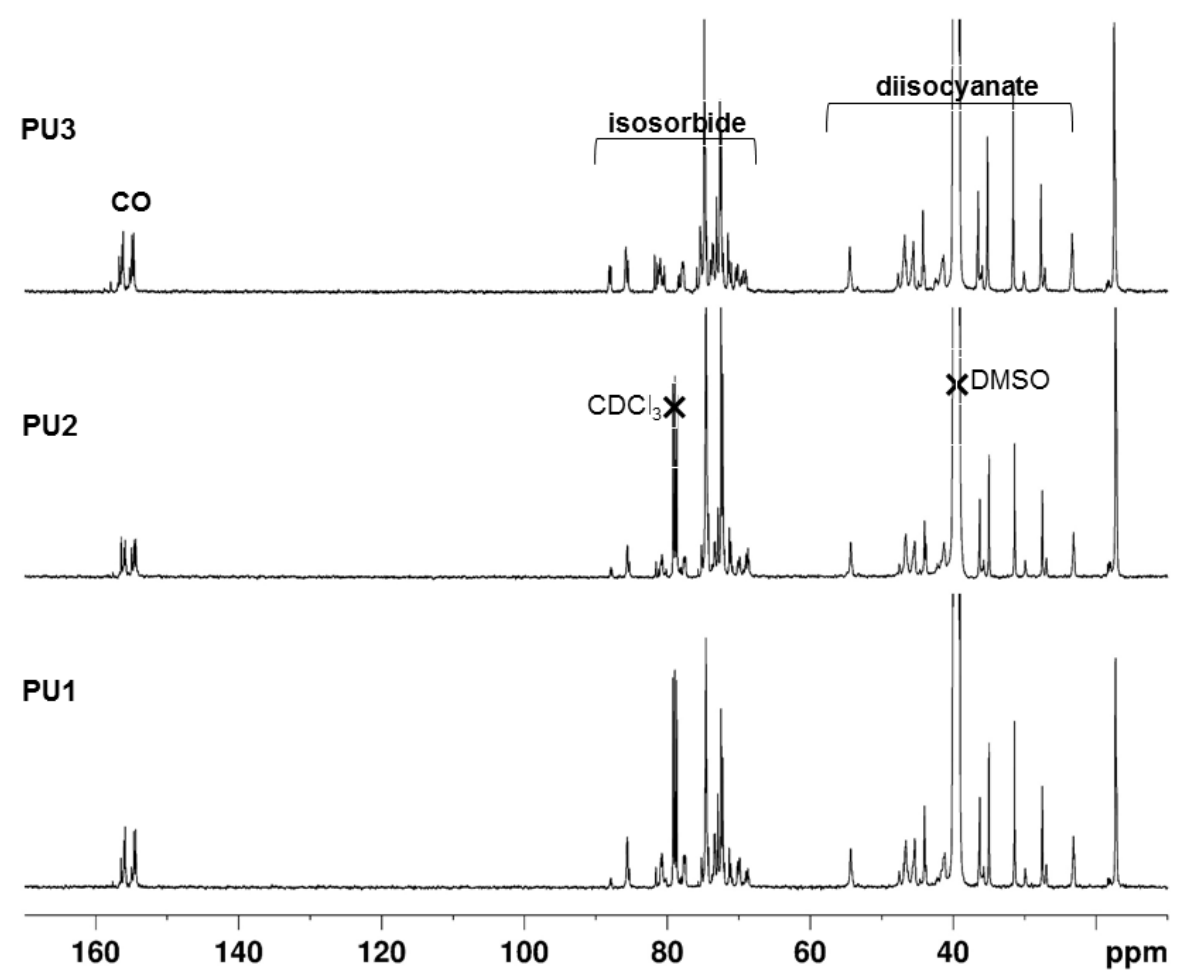

Figure 8. ${ }^{13} \mathrm{C}\left\{{ }^{1} \mathrm{H}\right\}$ NMR spectra of PU1, PU2 and PU3 in DMSO- $d_{6}$. Traces of $\mathrm{CDCl}_{3}$ are seen in the spectra of PU1 and PU2. 
ved between 65 and 90 ppm, while those of IPDI appear at higher field from 20 to $60 \mathrm{ppm}$. The carbonyl resonances appear between 153-157 ppm while the strong peaks centered at 72.4 and 74.6 ppm correspond to the methine and methylenic carbons of PPG segments, respectively. The methyl peaks appear at around $17.2 \mathrm{ppm}$. The experimental ${ }^{13} \mathrm{C}$ chemical shifts agree with those referenced in the literature [17] for pre-polymers made of PPG and IPDI monomers.

No peaks of the primary and secondary isocyanate groups of the IPDI terminal groups or monomers appear at 121.7 and $122.6 \mathrm{ppm}$ in the spectra [41], which indicates that all the IPDI monomers/units have reacted with the -OH groups of the diol monomers, in accordance with the FTIR results.

The ${ }^{13} \mathrm{C}\left\{{ }^{1} \mathrm{H}\right\}$ NMR spectrum of PU4 presented in Figure 9 is similar to those of the previous PUs, except that the higher field region is occupied by the peaks of HDMI monomers. HSQC and HMBC spectra were used to fully assign the in-chain carbons of PU4 (see Figures S9-S11 of the Supplementary Material). This similarity is found in the characterization of polyether urethane urea obtained by reaction between HMDI and polyethylene glycol and ethylenediamine [42].

The disappearance of the ${ }^{13} \mathrm{C}$ NMR peak at $124 \mathrm{ppm}$, characteristic of the isocyanate group, indicates that all the diisocyanate groups have fully reacted with the diol monomers [31]. This result was also confirmed by the FTIR analysis.

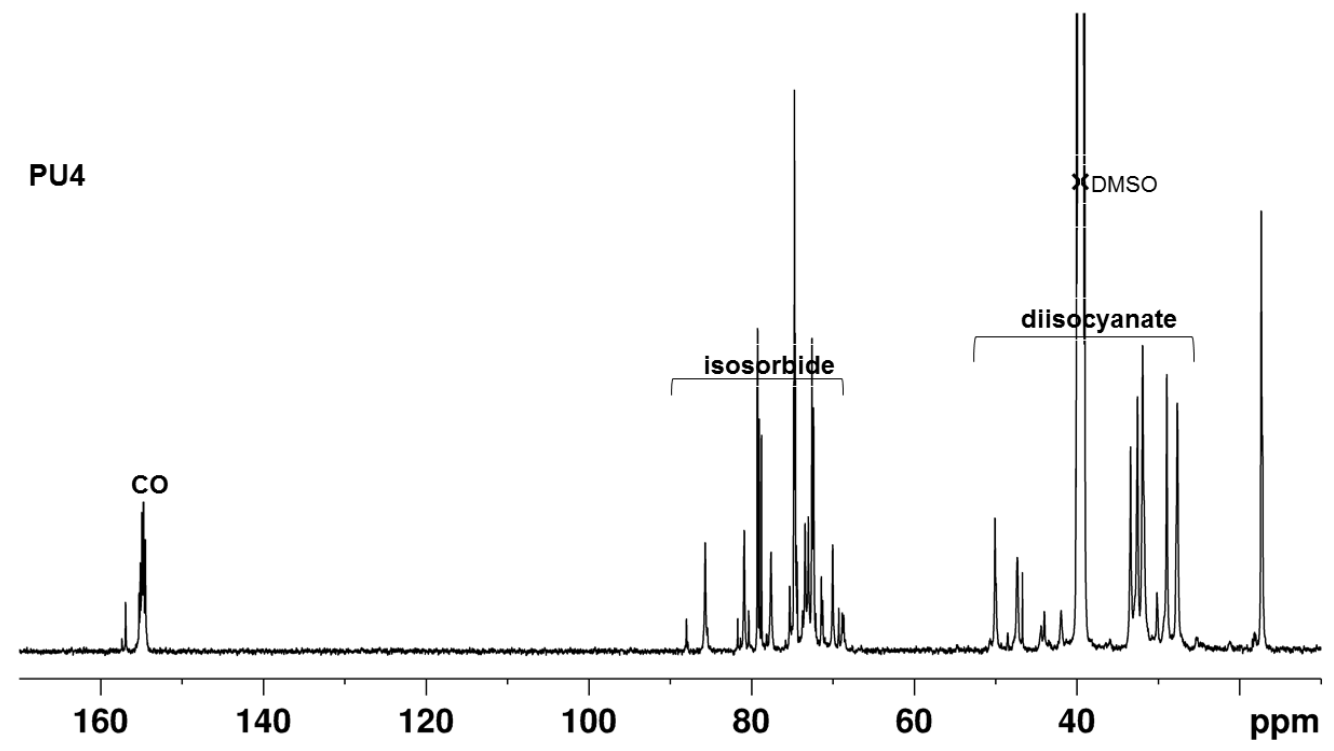

Figure 9. ${ }^{13} \mathrm{C}\left\{{ }^{1} \mathrm{H}\right\}$ NMR spectrum of the PU4 in DMSO- $d 6$.

\subsection{Determination of hydrodynamic radii of the PUs}

The determination of the diffusion coefficients of the PUs of this work, by the Pulse Gradient Spin Echo (PGSE) NMR method, enabled the calculation of the corresponding hydrodynamic radii $(\mathrm{rh})$ using the Stokes-Einstein equation for diluted solutions of large molecules [43]:

$$
\mathrm{D}_{0}=\frac{\mathrm{k}_{\mathrm{B}} \mathrm{T}}{6 \pi \mathrm{\eta} r_{h}}
$$

where $\mathrm{k}_{\mathrm{B}}$ is the Boltzmann's constant, $\mathrm{T}$ the temperature, $\eta$ the viscosity of the solution $\left(\mathrm{N} . \mathrm{s} . \mathrm{m}^{-2}=\mathrm{kg}\right.$. $\left.\mathrm{s}^{-1} \cdot \mathrm{m}^{-1}\right)$ and $\mathrm{rn}$ the hydrodynamic radius $(\mathrm{m})$. The viscosity of DMSO- $d_{6}$ at $30^{\circ} \mathrm{C}, \eta=1.951 \times 10^{-3}$ Pa.s, was estimated from that of the non-deuterated solvent taking into account the isotopic effects of the viscosity [44]. 
The self-diffusion coefficient $\mathrm{D}_{0}$ represents the translational movement of the solute at infinite dilution and, as shown by Equation 2, is dependent on the size and shape of the solute, temperature and viscosity of the solvent. The dependence of the diffusion coefficient D versus solute concentration was plotted for all the PUs (Figure S12 in Supplementary Material), being approximately linear for all polymers because the solutions are diluted. The self-diffusion coefficients $\mathrm{D}_{0}$ at infinite dilution $(\mathrm{c}=0)$ are summarized in Table 3 . The corresponding values of $\mathrm{rh}$ obtained from Equation 2 are also presented in Table 3.

In the family of polyurethanes based on IPDI diisocyanate (PU1, PU2 and PU3), the hydrodynamic radius increases with $\mathrm{M}_{\mathrm{n}}$ (see below in subsection 3.5.2.). When the IPDI is replaced by HMDI diisocyanate, as in the case of PU4, a substantial increase in the $\mathrm{rh}$ is observed in comparison with polymers with similar molecular weights, such as PU1 and PU3. The fragment derived from HMDI behaves like a larger (and more flexible) unit compared to IPDI, resulting in polymers of larger sizes for similar molar ratio of the monomers used in the synthesis of the linear polyurethanes.

Table 3. Measured average values of $D_{0}$ for the PUs determined by PGSE NMR and the corresponding hydrodynamic radii ( $\mathrm{rh})$ in DMSO- $d_{6}$, at $30{ }^{\circ} \mathrm{C}$.

\begin{tabular}{ccc}
\hline Polyurethane & $\mathrm{D}_{0 \times 10^{11}}\left(\mathrm{~m}^{2} / \mathrm{s}^{-1}\right)$ & $\mathrm{rh}(\mathrm{nm})$ \\
\hline PU1 & 7.04 & $1.62 \pm 0.04$ \\
PU2 & 4.49 & $2.65 \pm 0.15$ \\
PU3 & 6.74 & $1.69 \pm 0.04$ \\
PU4 & 4.51 & $2.52 \pm 0.19$ \\
\hline
\end{tabular}

\subsection{Determination of molecular weights of the PUs}

\subsubsection{Determination by GPC/SEC}

The highest number-average molecular weight was obtained for PU4, the lowest one corresponding to PU3 (Table 4), in agreement with the hydrodynamic radii of these polyurethanes. All the synthesized polyurethanes presented similar relatively narrow dispersities $\left(Đ=M_{w} / M_{n}\right)$.

Table 4. Average molecular weights and dispersities determined by GPC/SEC. ${ }^{a}$

\begin{tabular}{cccc}
\hline $\begin{array}{c}\text { Polyurethane } \\
\text { no. }\end{array}$ & $\begin{array}{c}\mathrm{M}_{\mathrm{w}} \mathrm{a} \\
\text { (Dalton) }\end{array}$ & $\begin{array}{c}\mathrm{M}_{\mathrm{n}} \mathrm{a} \\
\text { (Dalton) }\end{array}$ & $\begin{array}{c}Ð \\
\left(\mathrm{M}_{\mathrm{w}} / \mathrm{M}_{\mathrm{n}}\right)\end{array}$ \\
\hline PU1 & 15600 & 10300 & 1.50 \\
PU2 & 19200 & 12800 & 1.48 \\
PU3 & 9200 & 7200 & 1.27 \\
PU4 & 23500 & 15100 & 1.55 \\
\hline
\end{tabular}

a In polystyrene units.

Since the GPC/SEC measurements (see chromatograms in Figures S13 in Supplementary Material) were calibrated with polystyrene (PS) standards, the values of $\mathrm{Mn}_{\mathrm{n}}$ and $\mathrm{M}_{\mathrm{w}}$ are relative to PS and maybe quite different from the absolute values, because the hydrodynamic volumes of the present PUs are considerably different from those of polystyrenes with equivalent molecular weights.

\subsubsection{Determination by ${ }^{13} \mathrm{C}$ NMR spectroscopy}

Quantitative ${ }^{1} \mathrm{H}$ and ${ }^{13} \mathrm{C}$ NMR is a powerful tool to count monomer groups in homopolymers and copolymers of very well defined composition and narrow molecular weight distributions. The 
segmented linear polyurethanes herein described satisfy the above mentioned criteria as they present well defined linear chains, low molecular weights and dispersity indices below 1.5.

To improve the determination of $\mathrm{M}_{\mathrm{n}}$ values of the present polyurethanes and attempt to obtain absolute values, the area of methyl Ca of PPG segments, centered at $17.2 \mathrm{ppm}$, which corresponds to an average of 17 carbons per PPG segment, was used as reference instead of those of the copolymer end groups (see Tables S5-S8 of the Supplementary Material for composition calculations). The polyurethanes average compositions obtained in this way are shown in Table 5 together with the calculated $\mathrm{Mn}_{\mathrm{n}}$ values. These compositions agree with the monomer ratios used in the synthesis of polyurethanes PU1-PU4 (see Table 2).

The values obtained for $\mathrm{Mn}_{\mathrm{n}}$ calculated by NMR are substantially lower than those determined by GPC/SEC (see Table 4), meaning that the hydrodynamic volumes of these PUs are considerably higher than those of polystyrene standards with equivalent molar masses.

Table 5. Average compositions and $\mathrm{Mn}_{\mathrm{n}}$ of PU1-PU4 determined by NMR spectroscopy.

\begin{tabular}{cccccc}
\hline $\begin{array}{c}\text { Polyurethane } \\
\text { no. }\end{array}$ & $\begin{array}{c}\text { Number of } \\
\text { Isosorbide } \\
\text { units }{ }^{\text {a }}\end{array}$ & $\begin{array}{c}\text { Number of } \\
\text { IPDI } \\
\text { units }^{\text {a }}\end{array}$ & $\begin{array}{c}\text { Number of HDMI } \\
\text { units a }\end{array}$ & $\begin{array}{c}\text { Number } \\
\text { of PPG } \\
\text { segments }\end{array}$ & $\begin{array}{c}\text { Mn } \\
\text { (Dalton) }\end{array}$ \\
\hline PU1 & $5(\mathrm{t})$ & $6(\mathrm{t})$ & - & 1 & 3040 \\
PU2 & $5(\mathrm{t})$ & $7(\mathrm{t}, \mathrm{b})$ & - & 2 & 4270 \\
PU3 & $6(\mathrm{t})$ & 6 & - & 1 & 3210 \\
PU4 & $5(\mathrm{t})$ & - & $6(\mathrm{t})$ & 1 & 3380 \\
\hline
\end{tabular}

${ }^{a} \mathrm{t}=$ at least one monomer is terminal; $\mathrm{b}=$ one monomer is in between PPG segments.

${ }^{\mathrm{b}} \mathrm{Mn}$ values with uncertainties up to $15 \%$.

\subsection{Thermal properties of the PUs}

The evaluation of the thermal behavior of the samples (polymers) is crucial for biomedical applications. For instance, if the value of the glass transition temperature $\left(\mathrm{T}_{\mathrm{g}}\right)$ of the polymer is higher than the human body temperature the polymer is rigid (glass region). In contrast, if the $\mathrm{Tg}$ value is below the body temperature the sample is in the rubber or elastomeric state. Figure 10 displays the DSC thermograms of the four materials under study in the range -100 to $+230{ }^{\circ} \mathrm{C}$. The DSC results 


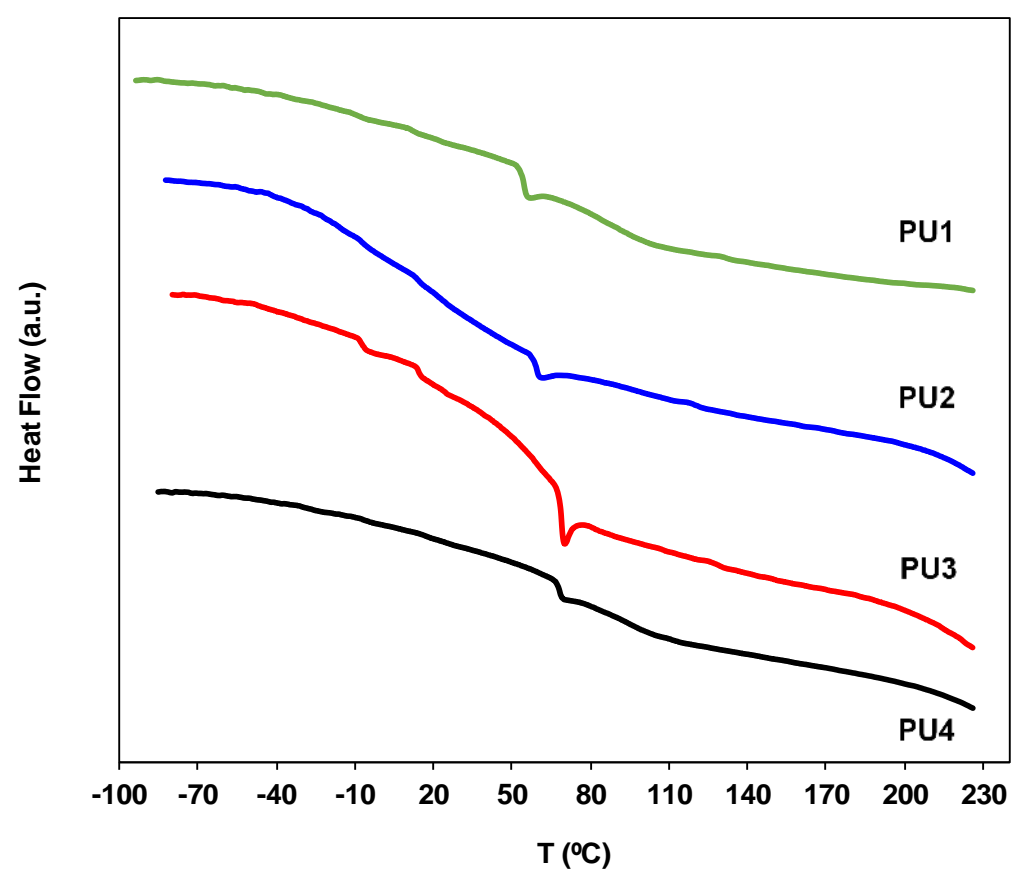

Figure 10. DSC thermograms of polyurethanes samples PU1-PU4 (exo up).

presented refer to the second thermal cycle and the $\mathrm{T}_{\mathrm{g}}$ value was determined as the onset point of the thermal event detected. All the samples are in the amorphous state and do not present phase separation. Thus a unique glass transition above the human body temperature is detectable, at 54.6, 57.7, 69.0 and $68.0{ }^{\circ} \mathrm{C}$, for the samples PU1 to PU4, respectively, which indicate that these polyurethanes exhibit a lacquer/varnish behavior. No other thermal events were detected in addition to these glass transition temperatures, except in the case of PU3 that exhibits two additional small $\mathrm{T}_{\mathrm{g}}$ events at lower temperatures $\left(-7.2\right.$ and $\left.14.4^{\circ} \mathrm{C}\right)$, with $\Delta \mathrm{C}_{\mathrm{p}}$ values $c a$. three times smaller. The PU3 sample has a higher content of low oligomers than the remaining samples, as can be observed in the corresponding GPC/SEC chromatograms (Figure S13 of the Supplementary Material), which can possibly give rise to very small domains characterized by lower $\mathrm{T}_{\mathrm{g}}$ values (likely corresponding to isolated short hard segments).

\subsection{Cytotoxicity assay}

The HaCaT keratinocyte cell line was selected to evaluate the cytotoxicity of the PUs synthesized in this work, since these cells are the predominant cell type in the adult human epidermis, the outermost layer of the skin, thus presenting an adequate in vitro cell model that better mimic in vivo conditions. The HaCaT cell viability, with 72 hours of exposure to polyurethanes PU1PU4, was evaluated by the MTT (3-(4,5-dimethylthiazol-2-yl)-2,5-diphenyltetrazolium bromide) reduction assay [45] (Figure 11). The tests corresponding to PU1-PU3 do not differ significantly from the control experiment (glass coverslip), whereas the cell viability for PU4 is $80 \% \pm 5 \%$ (i.e. significantly different from control for $\mathrm{p}<0.05$ ). 


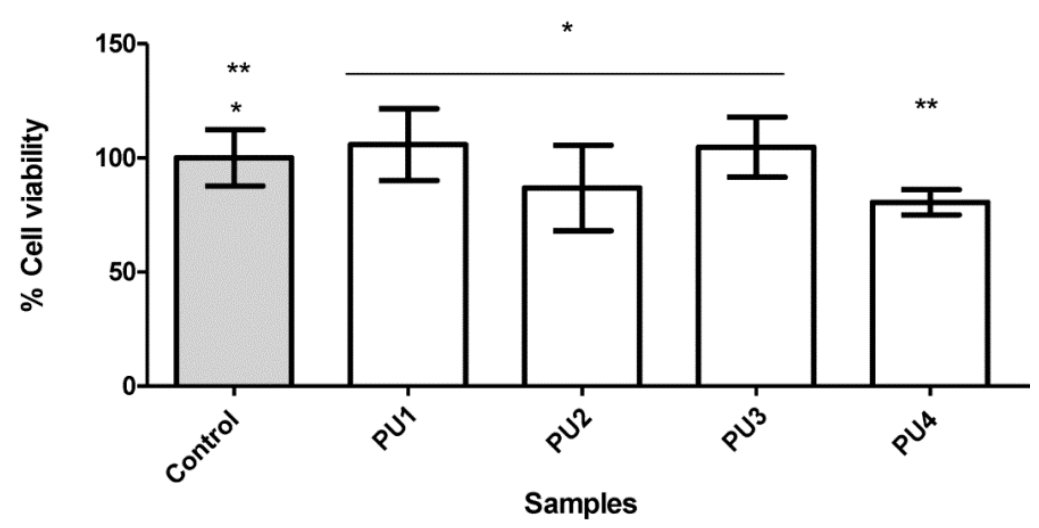

Figure 11. HaCaT cell viability by MTT after proliferation under polyurethanes PU1-PU4. Control (glass slide). (mean $\pm S D)(n=6) .\left({ }^{*}\right.$ Not significantly different $(p>0.05),{ }^{* *}$ significantly different $(p<$ $0.05)$ ).

The cell viability was higher than $70 \%$ for all the PUs tested. Thus, these materials can be considered biocompatible according to this in vitro assay. These results are in accordance with the previously published results, where the PU nail lacquers were evaluated and found to be material biocompatible, being proposed for nail applications as safe pharmaceutical excipients [11].

\section{Conclusions}

A convenient synthesis for polyurethanes from renewable sources is described. The structure of these polyurethanes was determined and confirmed by FTIR, ${ }^{1} \mathrm{H}$ and ${ }^{13} \mathrm{C}$ NMR spectroscopies of all four samples. The structures found confirm the higher reactivity of the secondary NCO group of IPDI compared to the primary group.

Quantitative ${ }^{13} \mathrm{C}$ NMR spectroscopy analysis enabled the calculation of the polyurethanes compositions, number-average molecular weights, as well as of their diffusion coefficients (D) and hydrodynamic radii $(\mathrm{r} h)$ from PGSE NMR experiments. Both values are in accordance with the molar ratio employed in the synthesis of the polyurethanes.

The polyurethanes were also characterized by GPC/SEC leading to molecular weights, which are considerably higher than the previous values, since they are determined relative to polystyrene standards, and relatively narrow molecular weight distributions, with dispersity indices $\mathrm{M}_{\mathrm{w}} / \mathrm{M}_{\mathrm{n}}<1.5$.

The DSC studies indicate that the four PU samples are in the amorphous state, being characterized by $\mathrm{T}_{\mathrm{g}}$ values above the human body temperature.

The polyurethanes obtained were biocompatible with keratinocyte cells in the conditions used in the tests. This is a relevant property that must be taken into account when considering pharmaceutical excipients. For this reason, these newly synthesized polymers can be used in pharmaceutics, namely as a component of nail lacquers for controlled-release drug delivery.

Supplementary Materials: The following are available online at www.mdpi.com/xxx/s1.

Author Contributions: Conceptualization, Pedro T. Gomes, Helena M. Ribeiro and João C. Bordado; Investigation, Barbara S. Gregorí Valdes, Clara S. B. Gomes, José R. Ascenso, Hermínio P. Diogo, Lídia M. Gonçalves and Rui Galhano dos Santos; Methodology, Pedro T. Gomes, José R. Ascenso, Helena M. Ribeiro and João C. Bordado; Project administration, Helena M. Ribeiro and João C. Bordado; Supervision, Pedro T. Gomes, Helena M. Ribeiro and João C. Bordado; Writing - original draft, Barbara S. Gregorí Valdes, Clara S. B. Gomes, Pedro T. Gomes, José R. Ascenso and Hermínio P. Diogo; Writing - review \& editing, Clara S. B. Gomes, Pedro T. Gomes and Helena M. Ribeiro.

Funding: The authors gratefully acknowledge the support of CERENA and CQE strategic projects (FCT-UID/ECI/04028/2013 and UID/QUI/00100/2013, respectively) funded by the Fundação para Ciência e a Tecnologia (FCT), Portugal. B.S.G.V., R.G.S. and C.S.B.G. also acknowledge FCT for fellowships (SFRH/BD/78962/2011, SFRH/BPD/105662/2015 and SFRH/BPD/107834/2015, respectively). 
Acknowledgments: We also thank to PTNMR network for the NMR facility.

Conflicts of Interest: The authors declare no conflict of interest.

\section{References}

[1] Sarkar, D.; Yang, J.C.; Sen Gupta, A.; Lopina, S.T. Synthesis and characterization of L-tyrosine based polyurethanes for biomaterial applications, J. Biomed. Mater. Res. - Part A., 2009, 90, 263-271, DOI:10.1002/jbm.a.32095.

[2] Han, J.; Chen, B.; Ye, L.; Zhang, A.Y.; Zhang, J.; Feng, Z.G. Synthesis and characterization of biodegradable polyurethane based on poly caprolactone and L-lysine ethyl ester diisocyanate. Front. Mater. Sci. China, 2009. 3, 25-32 DOI:10.1007/s11706-009-0013-4.

[3] Besse, V.; Auvergne, R.; Carlotti, S.; Boutevin, G.; Otazaghine, B.; Caillol, S.; Pascault, J.P.; Boutevin, B. Synthesis of isosorbide based polyurethanes: An isocyanate free method. React. Funct. Polym., 2013, 73, 588-594, DOI:10.1016/j.reactfunctpolym.2013.01.002.

[4] Chen, T.K.; Tien, Y.I.; Wei, K.H. Synthesis and characterization of novel segmented polyurethane clay nanocomposite via poly(epsilon-caprolactone)/clay. J. Polym. Sci. Part a-Polymer Chem., 1999, 37, 2225-2233. DOI:10.1002/(sici)1099-0518(19990701)37:13<2225::aid-pola37>3.0.co;2-z.

[5] Bachmann, F.; Reimer, J.; Ruppenstein, M.; Thiem, J. Synthesis of novel polyurethanes and polyureas by polyaddition reactions of dianhydrohexitol configurated diisocyanates, Macromol. Chem. Phys., 2001, 202, 3410-3419. DOI:10.1002/1521-3935(20011101)202:17<3410::AID-MACP3410>3.0.CO;2-Q.

[6] Guelcher, S.A.; Gallagher, K.M.; Didier, J.E.; Klinedinst, D.B.; Doctor, J.S.; Goldstein, A.S.; Wilkes, G.L.; Beckman, E.J.; Hollinger, J.O. Synthesis of biocompatible segmented polyurethanes from aliphatic diisocyanates and diurea diol chain extenders. Acta Biomater., 2005, 1, 471-484. DOI:10.1016/j.actbio.2005.02.007.

[7] Kim, H.-J.; Kang, M.-S.; Knowles, J.C.; Gong, M.S. Synthesis of highly elastic biocompatible polyurethanes based on bio-based isosorbide and poly(tetramethylene glycol) and their properties., J. Biomater. Appl., 2014, 29, 454-464, DOI:10.1177/0885328214533737.

[8] Abraham, G.A.; Marcos-Fernández, A.; San Román, J. Bioresorbable poly(ester-ether urethane)s from L-lysine diisocyanate and triblock copolymers with different hydrophilic character, J. Biomed. Mater. Res. Part A, 2006, 76, 729-736, DOI:10.1002/jbm.a.30540.

[9] Zhang, C.; Zhang, N.; Wen, X. Synthesis and characterization of biocompatible, degradable, light-curable, polyurethane-based elastic hydrogels, J. Biomed. Mater. Res. Part A, 2007, 82A, 637-650, DOI: 10.1002/jbm.a.30992.

[10] Spiridon, I.; Popa, V.I. Hemicelluloses: Major Sources, Properties and Applications. In Monomers, Polymers and Composites from Renewable Resources, M. N. Belgacem and A. Gandini (Eds.), 2008, ch. 13, pp. 289-304, Elsevier, DOI:10.1016/B978-0-08-045316-3.00013-2.

[11] Gregorí Valdes, B.S.; Serro, A.P.; Gordo, P.M.; Silva, A.; Gonçalves, L.; Salgado, A.; Marto, J.; Baltazar, D.; dos Santos, R.G.; Bordado, J.M.; Ribeiro, H.M. New Polyurethane Nail Lacquers for the Delivery of Terbinafine: Formulation and Antifungal Activity Evaluation, J. Pharm. Sci., 2017, 106, 1570-1577, DOI:10.1016/j.xphs.2017.02.017.

[12] Gogolewski, S.; Gorna, K.; Zaczynska, E.; Czarny, A. Structure-property relations and cytotoxicity of isosorbide-based biodegradable polyurethane scaffolds for tissue repair and regeneration, J. Biomed. Mater. Res. - Part A, 2008, 85, 456-465, DOI:10.1002/jbm.a.31481.

[13] Fenouillot, F.; Rousseau, A.; Colomines, G.; Saint-Loup, R.; Pascault, J.P. Polymers from renewable 1,4:3,6-dianhydrohexitols (isosorbide, isomannide and isoidide): A review. Prog. Polym. Sci., 2010, 35, 578622. DOI:10.1016/j.progpolymsci.2009.10.001.

[14] Stoss, P.; Hemmer, R. 1,4/3,6-Dianhydrohexitols. Adv. Carbohydr. Chem. Biochem., 1991, 49, 93-173, DOI:10.1016/S0065-2318(08)60182-1.

[15] Marques, M.F.F.; Gordo, P.M.; De Lima, A.P.; Queiroz, D.P.; De Pinho, M.N.; Major, P.; Kajcsos, Z. Free-volume studies in polycaprolactone/poly(propylene oxide) urethane/urea membranes by positron lifetime spectroscopy, in: Acta Phys. Pol. A, 2008, 113, 1359-1364, DOI:10.12693/APhysPolA.113.1359.

[16] Chattopadhyay, D.K.; Raju, N.P.; Vairamani, M.; Raju, K.V.S.N. Structural investigations of polypropylene glycol (PPG) and isophorone diisocyanate (IPDI) based polyurethane prepolymer by matrix-assisted laser 
desorption/ionization time-of-flight (MALDI-TOF)-mass spectrometry, Prog. Org. Coatings, 2008, 62, 117122. DOI:10.1016/j.porgcoat.2007.09.021.

[17] Prabhakar, A.; Chattopadhyay, D.K.; Jagadeesh, B.; Raju, K.V.S.N. Structural investigations of polypropylene glycol (PPG) and isophorone diisocyanate (IPDI)-based polyurethane prepolymer by 1D and 2D NMR spectroscopy, J. Polym. Sci. Part A Polym. Chem., 2005, 43, 1196-1209, DOI:10.1002/pola.20583.

[18] Lee, C.-H.; Takagi, H.; Okamoto, H.; Kato, M.; Usuki, A. Synthesis, Characterization, and Properties of Polyurethanes Containing 1,4:3,6-Dianhydro-D-sorbitol, J. Poly. Sci: Part A: Poly. Chem., 2009, 47, 60256031, DOI:10.1002/pola.23645.

[19] Marín, R.; Alla, A.; Ilarduya, A.M.; Munõz-Guerra, S.; Carbohydrate-Based Polyurethanes: Comparative Study of Polymers Made from Isosorbide and 1,4-Butanediol, J. Appl. Poly. Sci., 2012, 123, 986-994, DOI:10.1002/app.34545.

[20] Daniel-da-Silva, A.L.; Bordado, J.C.M.; Martín-Martínez, J.M. Moisture curing kinetics of isocyanate ended urethane quasi-prepolymers monitored by IR spectroscopy and DSC, J. Appl. Polym. Sci., 2008, 107, 700709, DOI:10.1002/app.26453.

[21] Gregorí Valdés, B.S.; Bordado, J.M.; Ribeiro, H.; Bello, A.; Fernández, M.; Fernandes, S.; Vázquez, NA.R. Analysis and Characterization of Quasi-Prepolymers Obtained from Polyethylene Glycol 1500 and 4,4'-Diphenylmethane-DiIsocyanate, Mater. Sci. Eng. Adv. Res., 2015, 1, 10-15, DOI:10.24218/msear.2015.08.

[22] Price, W.S.. Pulsed-Field Gradient Nuclear Magnetic Resonance as a Tool for Studying Translational Diffusion. Part 1. Basic Theory, Concepts Magn. Reson., 1997, 9, 299-336. DOI:10.1002/chin.199750339.

[23] Geil, B. Measurement of translational molecular diffusion using ultrahigh magnetic field gradient NMR, Concepts Magn. Reson., 1998, 299-321, DOI:10.1002/(SICI)1099-0534(1998)10:5<299::AID-CMR3>3.0.CO;2-S.

[24] Moura Ramos, J.J.; Taveira-Marques, R.; Diogo, H.P. Estimation of the fragility index of indomethacin by DSC using the heating and cooling rate dependency of the glass transition, J. Pharm. Sci., 2004, 93, 15031507, DOI: 10.1002/jps.20061.

[25] International Organization for Standardization, Biological Evaluation of Medical Devices Part 5: Tests for In Vitro Cytotoxicity, Iso 10993-5, 2009, 5, 1-52.

[26] Kuan, H.-C.; Chuang, W.-P.; Ma, C.-C.M.; Chiang, C.-L.; Wu, H.-L. Synthesis and characterization of a clay/waterborne polyurethane nanocomposite., J. Mater. Sci., 2005, 40, 179-185, DOI: 10.1007/s10853-005-5704-3.

[27] Ferreira, P.; Pereira, R.; Coelho, J.F.J.; Silva, A.F.M.; Gil, M.H. Modification of the biopolymer castor oil with free isocyanate groups to be applied as bioadhesive, Int. J. Biol. Macromol., 2007, 40, 144-152, DOI:10.1016/j.ijbiomac.2006.06.023.

[28] Ferreira, P.; Silva, A.F.M.; Pinto, M.I.; Gil, M.H. Development of a biodegradable bioadhesive containing urethane groups, J. Mater. Sci. Mater. Med., 2008, 19, 111-120, DOI:10.1007/s10856-007-3117-3.

[29] Li, G.; Li, P.; Qiu, H.; Li, D.; Su, M.; Xu, K. Synthesis, characterizations and biocompatibility of alternating block polyurethanes based on P3/4HB and PPG-PEG-PPG, J. Biomed. Mater. Res. Part A., 2011, 98A, 88-99, DOI:10.1002/jbm.a.33100.

[30] Alves, P.; Coelho, J.F.J.; Haack, J.; Rota, A.; Bruinink, A.; Gil, M.H. Surface modification and characterization of thermoplastic polyurethane, Eur. Polym. J., 2009, 45, 1412-1419, DOI:10.1016/j.eurpolymj.2009.02.011.

[31] Rahman, M.M.; Hasneen, A.; Chung, I.; Kim, H.; Lee, W.-K.; Chun, J.H. Synthesis and properties of polyurethane coatings: the effect of different types of soft segments and their ratios, Compos. Interfaces, 2013, 20, 15-26, DOI:10.1080/15685543.2013.762890.

[32] Caracciolo, P.C.; Buffa, F.; Abraham, G.A. Effect of the hard segment chemistry and structure on the thermal and mechanical properties of novel biomedical segmented poly(esterurethanes), J. Mater. Sci. Mater. Med., 2009, 20, 145-155, DOI:10.1007/s10856-008-3561-8.

[33] Kricheldorf, H.R.; Chatti, S.; Schwarz, G.; Krüger, R.P. Macrocycles 27: Cyclic Aliphatic Polyesters of Isosorbide, J. Polym. Sci. Part A Polym. Chem., 2003, 41, 3414-3424, DOI:10.1002/pola.10933.

[34] Ferreira, P.; Coelho, J.F.J.; Pereira, R.; Silva, A.F.M.; Gil, M.H. Synthesis and characterization of a poly(ethylene glycol) prepolymer to be applied as a bioadhesive, J. Appl. Polym. Sci., 2007, 105, 593-601, DOI:10.1002/app.26206. 
[35] Vieira, A.P.; Ferreira, P.; Coelho, J.F.; Gil, M.H. Photocrosslinkable Polymers for Biomedical Applications, Int. J. Biol. Macromol., 2008, 43, 325-332, DOI:10.1016/j.ijbiomac.2008.06.002.

[36] Ferreira, P.; Coelho, J.F.; Gil, M.H. Developmet of a new photocrosslinkable biodegradable bioadhesive., Int. J. Pharm., 2008, 352, 172-181, DOI: 10.1016/j.ijpharm.2007.10.026.

[37] Daniel da Silva, A.L.; Martín-Martinez, J.M.; Bordado, J.C.M. Influence of the storage of reactive urethane quasi-prepolymers in their compositions and adhesion properties., Int. J. Adhes. Adhes., 2007, 28, 29-37, DOI:10.1016/j.ijadhadh.2007.03.002.

[38] Zhu, R.; Wang, Y.; Zhang, Z.; Ma, D.; Wang, X. Synthesis of polycarbonate urethane elastomers and effects of the chemical structures on their thermal, mechanical and biocompatibility properties, Heliyon, 2016, 2, e00125, DOI:10.1016/j.heliyon.2016.e00125.

[39] Zhang, S.; Cheng, L.; Hu, J. NMR studies of water-borne polyurethanes, J. Appl. Polym. Sci., 2003, 90, 257260, DOI:10.1002/app.12696.

[40] Rahman, M.M.; Hasneen, A.; Jo, N.J.; Kim, H.I.; Lee, W.K. Properties of Waterborne Polyurethane Adhesives with Aliphatic and Aromatic Diisocyanates, J. Adhes. Sci. Technol., 2011, 25, 2051-2062, DOI:10.1163/016942410x544857.

[41] Götz, H.; Beginn, U.; Bartelink, C.F.; Grünbauer, H.J.M.; Möller, M. Preparation of isophorone diisocyanate terminated star polyethers, Macromol. Mater. Eng., 2002, 287, 223-230, DOI:10.1002/1439-2054(20020401)287:4<223:AID-MAME223>3.0.CO;2-Z.

[42] Wang, H.; Kao, H.; Digar, M.; Wen, T. FTIR and Solid State 13 C NMR Studies on the Interaction of Lithium Cations with Polyether Poly(urethane urea), Macromolecules, 2011, 34, 529-537, DOI:10.1021/ma001096k.

[43] Mondiot, F.; Loudet, J.C.; Mondain-Monval, O.; Snabre, P.; Vilquin, A.; Würger, A. Stokes-Einstein diffusion of colloids in nematics, Phys. Rev. E - Stat. Nonlinear, Soft Matter Phys., 2012, 86, 010401(R), doi:10.1103/PhysRevE.86.010401.

[44] Holz, M.; Mao, X.A.; Seiferling, D.; Sacco, A. Experimental study of dynamic isotope effects in molecular liquids: Detection of translation-rotation coupling, J. Chem. Phys., 1996, 104, 669-679, DOI:10.1063/1.470863.

[45] Mosmann, T. Rapid colorimetric assay for cellular growth and survival: application to proliferation and cytotoxicity assays, J. Immunol. Methods, 1983, 65, 55-63, DOI:10.1016/0022-1759(83)90303-4. 